\title{
Twin prime numbers and Diophantine equations Ibrahima GUEYE
} Keywords: Twin primes; Diophantine equations; Wilson's theorem; Clement's theorem and
corollary; Bachet-Bézout Theorem.

\begin{abstract}
For two millennia, the prime numbers have continued to fascinate mathematicians. Indeed, a conjecture which dates back to this period states that the number of twin primes is infinite. In 1949 Clement showed a theorem on twin primes. Starting from Wilson's theorem, Clement's theorem and the corollary of Clement's theorem [1], I came to find Diophantine equations whose solution could lead to the proof of the infinitude of twin primes.
\end{abstract}

\section{Introduction:}

Or $(\mathrm{p}, \mathrm{q})$ a pair of integers such that $\mathrm{p}$ and $\mathrm{q}$ are both prime and $\mathrm{p}<\mathrm{q}$. We say that $(\mathrm{p}, \mathrm{q})$ form a pair of twin primes if $q=p+2$.

The couple $(2,3)$ is the only pair of consecutive primes.

Omitting the pair $(2,3), 2$ is the smallest possible distance between two primes, twin primes are two and two consecutive odd numbers.

Any pair of twin primes (with the exception of the couple $(3,5))$ is of the form $(6 n-1,6 n+1)$ for some integer $\mathrm{n}$. Indeed, any set of three consecutive natural numbers has at least a multiple of 2 (possibly two) and one multiple of 3, these two are confused between multiple two twin primes [4]. It is possible to show that, for any integer, the pair $(\mathrm{m}, \mathrm{m}+2)$ consists of twin primes if and only if:

$$
2[(\mathrm{M}-1) !+1]+\mathrm{m}=0 \bmod \mathrm{m}(\mathrm{m}+2)
$$

This characterization of factorial and modular twin primes was discovered by P. A. Clement in 1949 [2].

The series of reciprocals of twin primes converges to Brun's constant, unlike the series of reciprocals of prime numbers. This property was demonstrated by Viggo Brun in 1919 [3].

The twin prime conjecture states that there are infinitely many twin primes. In other words, there are infinitely many primes $\mathrm{p}$ such that $(\mathrm{p}+2)$ is also prime.

In 1940, Paul Erdős proved the existence of a constant $\mathrm{c}<1$ and infinitely many primes $\mathrm{p}$ such that: $p^{\prime}-p<\operatorname{cln}(p)$ where $p^{\prime}$ denotes the number immediately following the first $p$.

This result was improved several times, in 1986, Helmut Maier showed a constant $\mathrm{c}<0.25$ could be reached.

In 2003, Daniel Goldston and Cem Yildirim have shown that, for all $\mathrm{c}>0$, there are infinitely many primes $p$ such that $\mathrm{p}^{\prime}-\mathrm{p}<\mathrm{c} \ln (\mathrm{p})$.

In 1966, Chen Jingrun demonstrated the existence of infinitely many primes $p$ such that $p+2$ is the product of at most two prime factors (such a number, product of at most two prime factors, 2 is said -almost-first).

His approach was that of the theory of the screen, he used to treat similarly the twin prime conjecture and Goldbach's Conjecture.

As for me I establish relationships between the twin primes and special Diophantine equations.

\section{Theorem 1: Theorem Wilson}

Statement: An integer $\mathrm{p}$ strictly greater than 1 , is a prime number if and only if divides $(\mathrm{p}-1) !+1$, that is to say if and only if: $(p-1) !+1=0(\bmod p)$ 
Theorem 2: Theorem Clement

For any integer, the pair $(m, m+2)$ consists of twin primes if $4[(m-1) !+1]+m=0 \bmod m(m+2)$

\section{Theorem 3: Corollary of Clement's theorem [1]}

For any integer, the pair $(m, m+2)$ consists of twin primes if: $m(m+2)$ divides $((m-2)(m-1)$ ! 2).

\section{Theorem 4: Bachet-Bézout Theorem}

Given two integers $p$ and $q$ nonzero, if $r$ is the GCD of $p$ and $q$ then there exist two integers $x$ and $y$ such that: $r=x * p+y^{*} q$

In particular, two integers $\mathrm{p}$ and $\mathrm{q}$ are coprime if and only if there exist two integers $\mathrm{x}$ and $\mathrm{y}$ such that $x^{*} p+y^{*} q=1$

\section{Conjecture about Diophantine equations and twin primes:}

Is the set of integers $n>4$ such that $n$ and $(n+2)$ are twin primes. Let $a, b, c$ and $d$ non-zero integers. The following three Diophantine equations admit infinitely many solutions.

$$
\begin{aligned}
& f(n)=a * d * M+3 * a * d * n^{2 *} n+(2 * a * d+2 * a-b * d) * n^{2}+(3 * a-2 * b * d-2 * a * b-b) * n+4 * a * b-2 * a=0 \\
& g(n)=a * c * M+a *(3 * c-1) * n^{2 *} n+(2 * a * c-b * c-5 * a) * n^{2}+(b-2 * b * c-4 * a) * n-8 * a * b+4 * a=0 \\
& h(n)=c^{*} n^{2}-(4 * d+1) * n-2 *(4 * d+1+2 * c)=0
\end{aligned}
$$

M means $n$ exponent 4 ;

\section{Attempt of proof:}

By Wilson's theorem $n$ is a prime number if it divides $((n-1) !+1)$. And $(n+2)$ is a prime prime number if it divides $((n+1) !+1)$. Consider all values of $n$ such that $n$ and $(n+2)$ are twin primes.

So there are two nonzero integers $a$ and $b$ such that:

$$
\begin{gathered}
a=((n-1) !+1) / n \text { and } b=((n+1) !+1) /(n+2) \\
b=\left(\left(n^{2}+n\right) *(n-1) !+1\right) /(n+2)
\end{gathered}
$$

So, $\quad \mathrm{n}=((\mathrm{n}-1) !+1) / \mathrm{a} ;(\mathrm{n}+2)=\left(\left(\mathrm{n}^{2}+\mathrm{n}\right) *(\mathrm{n}-1) !+1\right) / \mathrm{b}$

$(n+2)-n=\left(\left(n^{2}+n\right) *(n-1) !+1\right) / b-((n-1) !+1) / a=\left(\left(a^{*} n^{2}+a * n-b\right)(n-1) !+(a+b)\right) / a * b=2$

After calculations we arrive at:

$$
(n-1) !=(2 * a * b-(a-b)) /\left(a * n^{2}+a * n-b\right)
$$

By the theorem of Clement of 1949 and we know that $n(n+2)$ are twin primes if:

$$
n *(n+2) \text { divides }(4 *((n-1) !+1)+n) \text {. }
$$

So there exists an integer c such that $c=(4 *((n-1) !+1)+n) /(n *(n+2))(n-1) !=\left(c^{*} n^{*}(n+2)-n-4\right)$ 14

By the corollary of the theorem of Clement and $n^{*}(n+2)$ are twin primes if:

$$
n *(n+2) \text { divides }((n-2) *(n-1) !-2) \text {. }
$$


So there exists an integer $d$ such that $d=((n-2) *(n-1) !-2) /(n *(n+2))(n-1) !=(d * n *(n+2)+2) /$ $(\mathrm{n}-2)$

Hence,

$$
(n-1) !=(2 * a * b-(a-b)) /\left(a * n^{2}+a * n-b\right)=(c * n *(n+2)-n-4) / 4=(d * n *(n+2)+2) /(n-2)
$$

If we associate two to two, the three expressions we obtain the following three Diophantine equations:

$$
\begin{gathered}
f(n)=a * d * M+3 * a * d * n^{2 *} n+(2 * a * d+2 * a-b * d) * n^{2}+(3 * a-2 * b * d-2 * a * b-b) * n+4 * a * b-2 * a=0 \\
g(n)=a * c * M+a *(3 * c-1) * n^{2 *} n+(2 * a * c-b * c-5 * a) * n^{2}+(b-2 * b * c-4 * a) * n-8 * a * b+4 * a=0 \\
h(n)=c * n^{2}-(4 * d+1) * n-2 *(4 * d+1+2 * c)=0
\end{gathered}
$$

M means n exponent 4.

Easiest to solve is $\mathrm{h}(\mathrm{n})=\mathrm{c} * \mathrm{n}^{2}-(4 * \mathrm{~d}+1) * \mathrm{n}-2(4 * \mathrm{~d}+1+2 * \mathrm{c})=0$

I call it "The Diophantine equation of twin primes"

$\mathrm{c}$ and $\mathrm{d}$ are nonzero integers

$\mathrm{n}>4$ such that $\mathrm{n}$ and $(\mathrm{n}+2)$ are twin primes.

If we can demonstrate the existence of infinitely many solutions then we can conclude of the infinite of twin primes.

Solve the equation $\mathrm{h}(\mathrm{n})=\mathrm{c} * \mathrm{n}^{2}-(4 * \mathrm{~d}+1) * \mathrm{n}-2 *(4 * \mathrm{~d}+1+2 * \mathrm{c})=0$

Delta $=16 * \mathrm{~d}^{2}+32 * \mathrm{c} * \mathrm{~d}+16 * \mathrm{c}^{2}+8 * \mathrm{~d}+8 * \mathrm{c}+1=(4 * \mathrm{~d}+4 * \mathrm{c}+1)^{2}$

The only solution is $\mathrm{n}=(4 * \mathrm{~d}+2 * \mathrm{c}+1) / \mathrm{c}$, the other solution is necessarily negative.

$$
\begin{gathered}
\mathrm{n}=(4 * \mathrm{~d}+1) / \mathrm{c}+2 \\
(\mathrm{n}+2)=(4 * \mathrm{~d}+1) / \mathrm{c}+4
\end{gathered}
$$

Recall that the twin primes are of the form $(6 * \mathrm{~m}-1)$ and $(6 * \mathrm{~m}+1)$

Let $\mathrm{n}=(6 * \mathrm{~m}-1)$; So $(\mathrm{n}-2)=(4 * \mathrm{~d}+1) / \mathrm{c}=(6 * \mathrm{~m}-3)$

$\mathrm{m}=(4 * \mathrm{~d}+3 * \mathrm{c}+1) /(6 * \mathrm{c})$

Let $\mathrm{k}$ be a nonzero as entire: $\mathrm{k}=(4 * \mathrm{~d}+1) / \mathrm{c}$

$$
\begin{aligned}
& \mathrm{c}^{*} \mathrm{k}=4 * \mathrm{~d}+1 \\
& \mathrm{c} * \mathrm{k}-4 * \mathrm{~d}=1
\end{aligned}
$$

According to Bachet-Bézout theorem, $\mathrm{c}$ and $\mathrm{d}$ are coprime.

Example: The first solution is for $\mathrm{n}=5 ;(\mathrm{n}+2)=7 ; \mathrm{c}=3$ and $\mathrm{d}=2$; in same time we remark that $\mathrm{a}=5$ and $\mathrm{b}=103$

The second solution is for $\mathrm{n}=11 ;(\mathrm{n}+2)=13 ; \mathrm{c}=101505$ and $\mathrm{d}=228386$

\section{Generalization}

I think that characterization of Clement of 1949 is just a special case of a more comprehensive characterization of twin primes.

Now consider the set of integers $n>4$. I propose the following characterization simpler and more general about twin primes:

Consider the nonzero integers $d^{\prime}$ and $c^{\prime}$ and $m$ such that:

$$
\left(6^{*} m-1\right)=n=\left(4 d^{\prime}+1\right) / c^{\prime}+2 \text { and }\left(6^{*} m+1\right)=(n+2)=\left(4 d^{\prime}+1\right) / c^{\prime}+4
$$

I conjecture that for each twin primes we can find two primes c' and $d^{\prime}$. In this case $m=$ $\left(4 * d^{\prime}+3 * c^{\prime}+1\right) /\left(6 * c^{\prime}\right)$

Examples:

$\mathrm{d}^{\prime}=5$ and $\mathrm{c}^{\prime}=7 ; \mathrm{n}=5$ and $(\mathrm{n}+2)=7$

$\mathrm{d}^{\prime}=29$ and $\mathrm{c}^{\prime}=13 ; \mathrm{n}=11$ and $(\mathrm{n}+2)=13$

$d^{\prime}=11$ et $c^{\prime}=3 ; n=17$ and $(n+2)=19$

$d^{\prime}=47$ and $c^{\prime}=7$ we have $n=29$ and $(n+2)=31$

$d^{\prime}=29$ and $c^{\prime}=3$ we have $n=41$ and $(n+2)=43$ Etc... 


\section{References}

[1] Ibrahima GUEYE, A note on the twin primes, South Asian Journal of Mathematics, Volume 2 (2012) Issue 2, p. 159-161

[2] PA Clement, Congruences for sets of premiums, American Mathematical Monthly 56 (1949), p. 23-25

[3] Viggo Brun, Series 1/5+1/7+1/11+1/13+1/17+1/19+1/29+1/31+1/41+1/43+1/59+ $1 / 61+\ldots$ where denominators are "twin primes" is convergent or over, Bulletin of Mathematical Sciences 43 (1919), p. 100-104 and 124-128.

[4] http://fr.wikipedia.org/wiki/Nombres_premiers_jumeaux 